\title{
GALOIS COHOMOLOGY OF FINITELY GENERATED MODULES
}

\author{
Dedicated to Professor T. Tannaka on his 60th birthday
}

\author{
TOYOFUMI TAKAHASHI
}

(Received August 20, 1968)

The purpose of this paper is to generalize Tate's theorem concerning Galois cohomology of finite modules. It will be shown here that a large part of the theorem holds also for finitely generated modules. In section 2 we shall consider, particularly, unramified cohomology of finitely generated modules over local fields. In the final section, we shall study the relation between local and global cohomology. I wish to express my thanks to Dr. K. Uchida for his useful suggestions.

1. Notation. Let $R$ be a Dedekind ring with field of fractions $k$. Let $\Omega$ be the union of all finite extensions $K$ of $k$ in which the integral closure of $R$ is unramified over $R$, and let $\bar{R}$ denote the integral closure of $R$ in $\Omega$. Let $G_{R}$ denote the Galois group of the extension $\Omega / k$. For any discrete $G_{R}$-module $A$, we put

$$
\begin{aligned}
& H^{r}(R, A)=H^{r}\left(G_{R}, A\right) \quad(r \in \boldsymbol{Z}) \\
& \widehat{H}^{r}(R, A)= \begin{cases}H^{r}\left(G_{R}, A\right) & (r \geqq 1) \\
\widehat{H}^{r}\left(G_{R}, A\right) & (r \leqq 0)\end{cases}
\end{aligned}
$$

(cf. [5]). By $M$ we shall always understand a finitely generated discrete $G_{R}$-module such that the order of the torsion part of $M$ is invertible in $R$. Such a module $M$ is said to be a Galois module over $R$. We put $T=$ [the torsion part of $M], F=M / T$ and $M^{\prime}=\operatorname{Hom}\left(M, \bar{R}^{\times}\right)$where $\bar{R}^{\times}$is the group of units of $\bar{R}$. For any locally compact abelian group $H$, we let $H^{*}$ denote its Pontrjagin character group.

2. Local fields. Let $k$ be a local field (i.e., a non-discrete locally compact field). Then we have isomorphisms

$$
\widehat{H}^{r}(k, M) \cong \widehat{H}^{2-r}\left(k, M^{\prime}\right)^{*}
$$


for all $r \in \boldsymbol{Z}$ (cf. [2; Chap. II, Théorème 6]). Suppose $k$ is a non-archimedean local field with valuation ring $\mathfrak{o}$. Let $M$ be a Galois module over $\mathfrak{o}$.

THEOREM 1. i) $H^{r}\left(\mathfrak{o}, M^{\prime}\right)=0 \quad(r \geqq 2)$.

ii) The inflation map $H^{2}(\mathrm{o}, M) \rightarrow H^{2}(k, M)$ and the canonical homomorphism $H^{0}\left(\mathfrak{o}, M^{\prime}\right) \rightarrow \widehat{H}^{\circ}\left(k, M^{\prime}\right)$ are injective. The subgroups $H^{2}(\mathfrak{o}, M)$ of $H^{2}(k, M)$ and $H^{\circ}\left(\mathfrak{o}, M^{\prime}\right)$ of $\widehat{H}^{\circ}\left(k, M^{\prime}\right)$ are the exact annihilators of each other.

iii) The inflation map $H^{1}(0, M) \rightarrow H^{1}(k, M)$ and the homomorphism $H^{1}\left(\mathfrak{o}, M^{\prime}\right) \rightarrow H^{1}\left(k, M^{\prime}\right)$ by the inflation map and the injection $\overline{\mathfrak{D}^{\times}} \rightarrow \overline{k^{x}}$ are injective. The subgroups $H^{1}(\mathfrak{o}, M)$ of $H^{1}(k, M)$ and $H^{1}\left(\mathfrak{o}, M^{\prime}\right)$ of $H^{1}\left(k, M^{\prime}\right)$ are the exact annihilators of each other.

ProOF. i) Since $\operatorname{cd} G_{3}=1$, we have $H^{r}\left(\mathfrak{o}, T^{\prime}\right)=0$ for $r \geqq 2$, and $H^{r}\left(\mathfrak{o}, M^{\prime}\right)=0$ for $r \geqq 3$. Since $\overline{\mathfrak{o}}^{x}$ is cohomologically trivial, we have $H^{r}\left(\mathfrak{o}, F^{\prime}\right)$ $=0$ for $r \geqq 1$. By the exact sequence

$$
H^{2}\left(\mathfrak{o}, F^{\prime}\right) \rightarrow H^{2}\left(\mathfrak{o}, M^{\prime}\right) \rightarrow H^{2}\left(\mathfrak{o}, T^{\prime}\right)
$$

we get $H^{2}\left(\mathfrak{o}, M^{\prime}\right)=0$.

ii) Consider a commutative diagram :

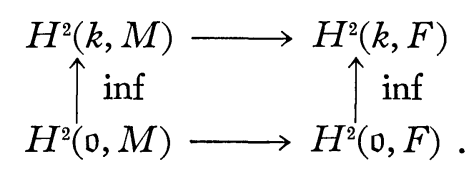

Since $H^{2}(\mathfrak{o}, M)$ is isomorphic to $H^{2}(\mathfrak{o}, F)$ and the inflation map $H^{2}(\mathfrak{o}, F)$ $\rightarrow H^{2}(k, F)$ is injective, the inflation map $H^{2}(\mathfrak{o}, M) \rightarrow H^{2}(k, M)$ is injective. For any finite extension $K$ of $k$, let $\widehat{K}^{\times}$denote the compactification of $K^{\times}$, and we put $\overline{k^{\star}}{ }^{\wedge}=\bigcup_{K} \widehat{K}^{\times}$, the union taken over all finite separable extensions $K$ of $k$. The injectivity of the map $H^{0}\left(\mathfrak{o}, M^{\prime}\right) \rightarrow \widehat{H}^{0}\left(k, M^{\prime}\right)$ is an immediate consequence of the fact $\hat{H}^{0}\left(k, M^{\prime}\right)=H^{0}\left(k, \operatorname{Hom}\left(M, \bar{k}^{\times \wedge}\right)\right)$. Let $k_{n r}$ denote the maximal unramified extension of $k$. Now by the $G$-split exact sequence

$$
0 \longrightarrow \overline{\mathfrak{o}}^{\times} \longrightarrow{\widehat{k_{n r}}}^{\mathrm{C}} \longrightarrow \widehat{\boldsymbol{Z}} \longrightarrow 0
$$

and $H^{\circ}\left(\mathfrak{o}, \operatorname{Hom}\left(M, \widehat{k}_{n r}^{\times}\right)\right)=H^{0}\left(k, \operatorname{Hom}\left(M, \bar{k}^{\times \wedge}\right)\right)$, we get an exact sequence

$$
0 \longrightarrow H^{0}\left(\mathfrak{o}, M^{\prime}\right) \longrightarrow H^{0}\left(k, \operatorname{Hom}\left(M, \bar{k}^{\times \wedge}\right)\right) \longrightarrow H^{0}(\mathfrak{o}, \operatorname{Hom}(M, \widehat{Z})) \longrightarrow 0 \text {. }
$$


Hence we get an exact sequence

$$
0 \longrightarrow H^{0}\left(\mathfrak{o}, M^{\prime}\right) \longrightarrow \widehat{H}^{0}\left(k, M^{\prime}\right) \longrightarrow H^{2}(\mathfrak{o}, M)^{*} \longrightarrow 0,
$$

because $\widehat{\boldsymbol{Z}}$ is a "module dualisant" for the group $G_{\mathfrak{v}}(\cong \widehat{\boldsymbol{Z}})$ (cf. [2; Chap. I, Annexe]).

iii) Consider a commutative diagram with exact rows

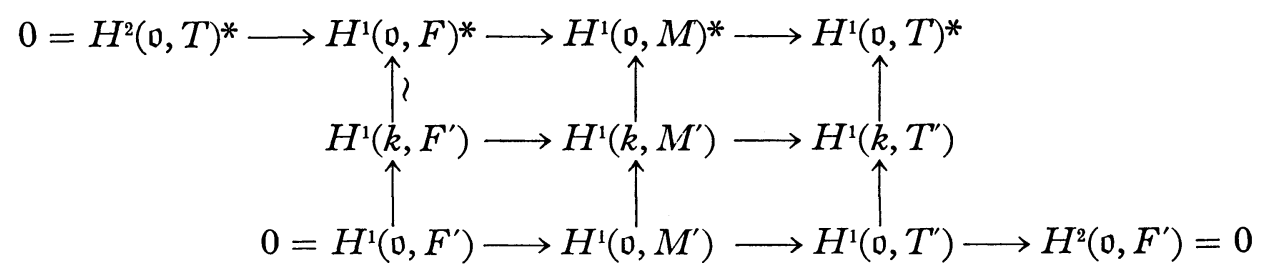

where $H^{1}\left(k, F^{\prime}\right)\left(\cong H^{1}(k, F)^{*}\right) \cong H^{1}(\mathfrak{o}, F)^{*}$. The sequence $H^{1}\left(\mathfrak{o}, T^{\prime}\right) \rightarrow H^{1}\left(k, T^{\prime}\right)$ $\rightarrow H^{1}(\mathfrak{o}, T)^{*}$ is exact by [3; Theorem 2.4]. Now it is easily verified that the sequence

$$
0 \longrightarrow H^{1}\left(\mathfrak{o}, M^{\prime}\right) \longrightarrow H^{1}\left(k, M^{\prime}\right) \longrightarrow H^{1}(\mathfrak{o}, M)^{*} \longrightarrow 0
$$

is exact and the theorem is proved.

3. Global fields. Let $k$ be a finite extension of $\boldsymbol{Q}$, or a function field in one variable over a finite field, let $S$ be a non-empty set of primes of $k$, including the archimedean ones, and $k_{S}$ denote the ring of elements in $k$ which are integers at all primes not in $S$. For each prime $v$ in $S$, let $k_{v}$ denote the completion of $k$ at $v$. Throughout this section, $M$ will be a Galois module over $k_{s}$. Let $P^{r}\left(k_{S}, M\right)$ (resp. $P^{r}\left(k_{S}, M^{\prime}\right)$ ) be the restricted direct product of $H^{r}\left(k_{v}, M\right)$ (resp. $H^{r}\left(k_{v}, M^{\prime}\right)(v \in S)$ relative to the subgroups $H^{r}\left(\mathfrak{o}_{v}, M\right)$ (resp. $\left.H^{r}\left(\mathfrak{o}_{v}, M^{\prime}\right)\right)$. Since $H^{1}\left(\mathfrak{o}_{v}, M\right)$ and $H^{1}\left(\mathfrak{o}_{v}, M^{\prime}\right)$ are finite, $P^{1}\left(k_{S}, M\right)$ and $P^{1}\left(k_{S}, M^{\prime}\right)$ are locally compact. By Theorem $1, P^{r}\left(k_{S}, M^{\prime}\right)$ is the direct sum for $r \geqq 2$. Since scd $G_{k_{v}}=2$ if $v$ is non-archimedean, $P^{r}\left(k_{S}, M\right)$ and $P^{r}\left(k_{S}, M^{\prime}\right)$ are equal to $\prod_{v \text { arch }} H^{r}\left(k_{v}, M\right)$ and $\prod_{v \text { arch }} H^{r}\left(k_{v}, M^{\prime}\right)$ respectively for $r \geqq 3$. The localization maps $H^{r}\left(k_{S}, M\right) \rightarrow H^{r}\left(k_{v}, M\right)$ and $H^{r}\left(k_{S}, M^{\prime}\right) \rightarrow H^{r}\left(k_{v}, M^{\prime}\right)$ give canonical maps :

$$
\begin{aligned}
& f_{r}: H^{r}\left(k_{S}, M\right) \longrightarrow P^{r}\left(k_{S}, M\right), \\
& f_{r}^{\prime}: H^{r}\left(k_{S}, M^{\prime}\right) \longrightarrow P^{r}\left(k_{S}, M^{\prime}\right) .
\end{aligned}
$$

By Theorem 1, local duality yields an isomorphism 


$$
P^{1}\left(k_{S}, M\right) \cong P^{1}\left(k_{S}, M^{\prime}\right)^{*} .
$$

Hence by duality we obtain maps :

$$
\begin{aligned}
& f_{1}^{*}: P^{1}\left(k_{S}, M^{\prime}\right) \longrightarrow H^{1}\left(k_{S}, M\right)^{*}, \\
& f_{1}^{\prime *}: P^{1}\left(k_{S}, M\right) \longrightarrow H^{1}\left(k_{S}, M^{\prime}\right)^{*} .
\end{aligned}
$$

Let $\Omega$ be the maximal extension of $k$ unramified outside $S$, and let $G$ be the Galois group of the extension $\Omega / k$. Let $J$ denote the projection to $S$ of the idèle group of $\Omega$, and we put $C=J / \overline{k_{S}^{\star}}$. Then $C$ is a class formation for extensions of $k$ unramified outside $S$. For simplicity, we put $J(M)$ $=\operatorname{Hom}(M, J)$ and $C(M)=\operatorname{Hom}(M, C)$. Let $l$ be a prime number such that $l k_{S}=k_{S}$.

\section{LEMMA $1 . \quad H^{r}\left(k_{S}, C(F)\right)(l)=0 \quad(r \geqq 3)$.}

PROOF. By Nakayama-Tate's Theorem, we have a commutative diagram whose horizontal arrows are isomorphisms

$$
\begin{gathered}
H^{r-2}\left(L / k, \underset{\hat{H}}{\operatorname{Hom}(F, \boldsymbol{Z}))} \stackrel{\sim}{\longrightarrow} H^{r}\left(L / k, \operatorname{Hom}(F, \boldsymbol{Z}) \otimes H^{0}\left(L_{S}, C\right)\right)\right. \\
\prod_{\uparrow}[L: K] \inf \\
H^{r-2}(K / k, \operatorname{Hom}(F, \boldsymbol{Z})) \stackrel{\inf }{\longrightarrow} H^{r}\left(K / k, \operatorname{Hom}(F, \boldsymbol{Z}) \otimes H^{0}\left(K_{S}, C\right)\right)
\end{gathered}
$$

for $r \geqq 3$, where $L \supset K$ are sufficiently large Galois extensions of $k$ unramified outside $S$. Since $l^{\infty} \mid[\Omega: k]$, we obtain $H^{r}\left(k_{s}, C(F)\right)(l)=H^{r}\left(k_{S}, \operatorname{Hom}(F, \boldsymbol{Z}) \otimes C\right)(l)$ $=\underset{\boldsymbol{K}}{\lim } H^{r}\left(K / k, \operatorname{Hom}(F, \boldsymbol{Z}) \otimes H^{0}\left(K_{S}, C\right)\right)(l)=0$.

Lemma 2. i) $\quad H^{1}\left(k_{S}, J(F)\right)=P^{1}\left(k_{s}, F^{v}\right)$.

ii)

$$
H^{r}\left(k_{S}, J(F)\right)(l)=P^{r}\left(k_{S}, F^{\prime}\right)(l) \quad(r \geqq 2) .
$$

PROOF. By Shapiro's Lemma, we have

$$
H^{r}\left(k_{S}, J(F)\right)=\sum_{v \in S} H^{r}\left(G_{v}, \operatorname{Hom}\left(F, \Omega_{v}^{\times}\right)\right) \quad(r \geqq 1)
$$

where $G_{v}$ is the decomposition subgroup for a place lying above $v$ and $\Omega_{v}$ is the extension of $k_{v}$ corresponding to $G_{v}$. We remark $P^{r}\left(k_{S}, F^{\prime}\right)$ is the direct sum for $r \geqq 1$. Of course, if $v$ is archimedean, $G_{v}=G_{k_{v}}$.

i) Let $v$ be a non-archimedean prime in $S$. Consider the inflationrestriction sequence: 


$$
0 \longrightarrow H^{1}\left(G_{v}, \operatorname{Hom}\left(F, \Omega_{v}^{\times}\right)\right) \longrightarrow H^{1}\left(k_{v}, F^{\prime}\right) \longrightarrow H^{1}\left(\Omega_{v}, F^{\prime}\right) \text {. }
$$

Since $G_{\Omega_{v}}$ acts trivially on $F$, we get $H^{1}\left(\Omega_{v}, F^{v}\right)=0$, hence

$$
H^{1}\left(G_{v}, \operatorname{Hom}\left(F, \Omega_{v}^{\times}\right)\right)=H^{1}\left(k_{v}, F^{\prime}\right) .
$$

ii) Let $v$ be non-archimedean. Since $l^{\infty} \mid\left[\Omega_{v}: k_{v}\right]$ and $G_{\Omega_{v}}$ acts trivially on $F$, we get $H^{2}\left(\Omega_{v}, F^{\prime}\right)(l)=0$. Since scd $G_{k_{v}}=2$, we get $H^{r}\left(\Omega_{v}, F^{\prime}\right)=0$ for $r \geqq 3$. Hence we obtain

$$
H^{r}\left(G_{v}, \operatorname{Hom}\left(F, \Omega_{v}^{\times}\right)\right)(l)=H^{r}\left(k_{v}, F^{v}\right)(l) \quad(r \geqq 2)
$$

by the inflation-restriction sequences.

Q.E.D.

THEOREM 2. Let $l$ be a prime number such that $l k_{S}=k_{S}$. Then

$$
f_{r}^{\prime}: H^{r}\left(k_{S}, M^{\prime}\right)(l) \cong \prod_{v \text { arch }} H^{r}\left(k_{v}, M^{\prime}\right)(l) \quad(r \geqq 3)
$$

ProOF. a) Consider an exact sequence :

$$
H^{r-1}\left(k_{S}, C(F)\right) \longrightarrow H^{r}\left(k_{S}, F^{v}\right) \longrightarrow H^{r}\left(k_{S}, J(F)\right) \longrightarrow H^{r}\left(k_{S}, C(F)\right) \text {. }
$$

By Lemmas 1 and 2 , we get the theorem in case $M=F$ and $r \geqq 4$.

b) Consider a commutative exact diagram :

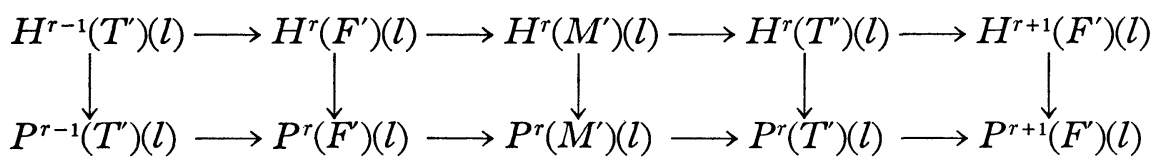

for $r \geqq 4$, where $H^{i}(\quad)=H^{i}\left(k_{S}, \quad\right)$ and $P^{i}(\quad)=P^{i}\left(k_{S}, \quad\right)$. By a) and $[3$; Theorem $3.1(c)$ ], each vertical map except the middle is isomorphic. Hence by Five Lemma the middle is also isomorphic.

c) Finally we must prove the theorem for $r=3$. We can find an open subgroup $U$ of $G$ such that its invariant field $K$ is totally imaginary and $M^{U}=M$. We have an exact sequence :

$$
0 \longrightarrow M \longrightarrow Q \longrightarrow A \longrightarrow 0
$$

where $Q$ is the induced module $\mathfrak{M}_{G}^{U}(M)$ (cf. [2; Chap. I, $\left.\mathrm{n}^{0} 2.5\right]$ ) and $A=Q / M$. Consider a commutative exact diagram : 


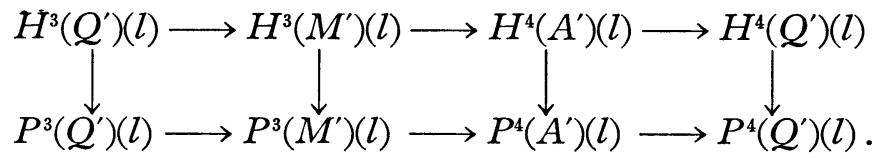

By Shapiro's Lemma, we have $H^{r}\left(k_{S}, Q^{\prime}\right)=H^{r}\left(K_{S}, M^{\prime}\right)$ and $P^{r}\left(k_{S}, Q^{\prime}\right)$ $=P^{r}\left(K_{S}, M^{\prime}\right)$. Since $K$ is totally imaginary, $P^{r}\left(K_{S}, M^{\prime}\right)=0$ for $r \geqq 3$. On the other hand, we have $H^{r}\left(K_{S}, F^{\prime}\right)(l)=0$ for $r \geqq 3$, because $G_{K_{S}}(=U)$ acts trivially on $F$ and $H^{r}\left(K_{S}, \bar{K}_{S}^{\times}\right)(l)=0$ for $r \geqq 3$. Hence we have $H^{r}\left(K_{S}, M^{\prime}\right)(l)$ $=H^{r}\left(K_{S}, T^{\prime}\right)(l)$ for $r \geqq 3$. Since $\operatorname{cd}_{l} G_{K_{S}}=2, H^{r}\left(K_{S}, T^{\prime}\right)(l)=0$ for $r \geqq 3$. Thus we get $H^{3}\left(k_{S}, M^{\prime}\right)(l) \cong P^{3}\left(k_{S}, M^{\prime}\right)(l)$ by the above diagram.

Q.E.D.

REMARK 1. The proof of Tate's Theorem [3; Theorem 3.1 (c)] which has been used in the above proof has been unpublished. It can be proved as follows: In the exact sequence $0 \rightarrow T^{\prime} \rightarrow J(T) \rightarrow C(T)$, the universal norms of $J(T)$ are mapped isomorphically onto the universal norms of $C(T)$. Hence we get an exact sequence : $\widehat{H}^{-1}\left(k_{S}, T^{\prime}\right) \rightarrow \widehat{H}^{-1}\left(k_{S}, J(T)\right) \rightarrow \widehat{H}^{-1}\left(k_{S}, C(T)\right) \rightarrow \widehat{H}^{0}\left(k_{S}, T^{\prime}\right)$ $\rightarrow \widehat{H}^{0}\left(k_{S}, J(T)\right)$ (cf. [5]). Since $T^{\prime}$ has no universal norms, $\widehat{H}^{-1}\left(k_{S}, T^{\prime}\right)=0$. It is easily shown that $\widehat{H}^{0}\left(k_{S}, T^{\prime}\right) \rightarrow \widehat{H}^{0}\left(k_{S}, J(T)\right)$ is injective, and $\widehat{H}^{-1}\left(k_{S}, J(T)\right)$ $=\prod_{v \text { a r } h \mathrm{~h}} \widehat{H}^{-1}\left(k_{v}, T^{\prime}\right)$. Hence we get $\widehat{H}^{3}\left(k_{S}, T\right) \cong \widehat{H}^{-1}\left(k_{S}, C(T)\right)^{*} \cong \widehat{H}^{-1}\left(k_{S}, J(T)\right)^{*}$ $\cong \prod_{v \text { arch }} \widehat{H}^{-1}\left(k_{v}, T^{\prime}\right)^{*} \cong \prod_{v \text { arch }} H^{3}\left(k_{v}, T\right)$. Let $K, Q$ and $A$ be as in the proof of Theorem 2 respectively, and $M=T$. Then $Q$ and $A$ are also finite. Consider a commutative exact diagram:

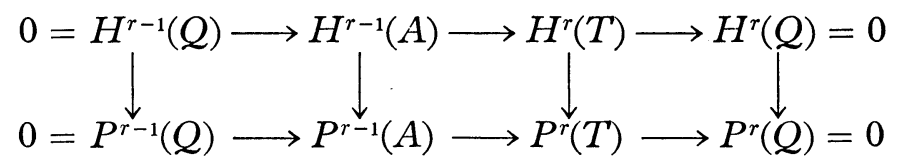

for $r \geqq 4$. By induction we get the theorem.

LEMMA 3. Suppose that there exists an open subgroup of $G$ which has strict cohomological dimension 2 for $l$. Then

$$
f_{r}: H^{r}\left(k_{S}, M\right)(l) \cong P^{r}\left(k_{S}, M\right)(l) \quad(r \geqq 3) .
$$

PROOF. By Theorem 2 we obtain $H^{r}\left(k_{S}, N\right)(l)=P^{r}\left(k_{S}, N\right)(l)(r \geqq 3)$ for any module $N$ of torsion. Using the exact sequence: $0 \rightarrow F \otimes \boldsymbol{Z}_{l} \rightarrow F \otimes \boldsymbol{Q}_{l}$ $\rightarrow F \otimes \boldsymbol{Q}_{l} / \boldsymbol{Z}_{l} \rightarrow 0$ and the above isomorphism, we get $H^{r}\left(k_{S}, F\right)(l) \cong P^{r}\left(k_{S}, F\right)(l)$ 
for $r \geqq 4$. Now the lemma can be proved similarly as Theorem 2 and Remark 1 .

THEOREM 3. i) If $k$ is a number field, then we have

$$
f_{r}: H^{r}(k, M) \cong \prod_{v \text { arch }} H^{r}\left(k_{v}, M\right) \quad(r \geqq 3) .
$$

ii) If $k$ is a function field, then we have

$$
H^{r}\left(k_{S}, M\right)=0 \quad(r \geqq 3) .
$$

PROOF. i) It is well known that $\operatorname{scd}_{l} G_{k}=2$ if $k$ is totally imaginary (in case $l=2$ ). ii) If $k$ is a function field, $C$ has no universal norm. Hence $\operatorname{scd} G_{k_{S}}=2$.

REMARK 2. In general case, Tate [3] has asserted that the group $G_{k_{S}}$ has strict cohomological dimension 2 for $l$ such that $l k_{S}=k_{S}$, except if $l=2$ and $k$ is not totally imaginary (the proof still remains unpublished).

THEOREM 4. $\operatorname{Im} f_{1}$ and $\operatorname{Im} f_{1}^{\prime}$ are the exact annihilators of each other in our duality (*). That is, the sequence

$$
H^{1}\left(k_{S}, M\right) \stackrel{f_{1}}{\longrightarrow} P^{1}\left(k_{S}, M\right) \stackrel{f_{1}^{\prime *}}{\longrightarrow} H^{1}\left(k_{S}, M^{\prime}\right)^{*}
$$

is exact.

Proof. a) In case $M=T$, the theorem was obtained by Tate [3; Theorem $3.1(\mathrm{~b})]$. We give here an outline of the proof:

For finite $S$, one can prove the equality

$$
\frac{\left[H^{0}\left(k_{S}, T\right)\right]\left[H^{2}\left(k_{S}, T\right)\right]}{\left[H^{1}\left(k_{S}, T\right)\right]}=\prod_{v \operatorname{arch}} \frac{\left[H^{0}\left(k_{S}, T\right)\right]}{|[T]|_{v}}
$$

by using Theorem 2 (cf. [4]). We have two exact sequences :

$$
\begin{gathered}
0 \longrightarrow H^{0}\left(k_{S}, T\right) \longrightarrow \prod_{v \in S} \widehat{H}^{0}\left(k_{v}, T\right) \longrightarrow H^{2}\left(k_{S}, T^{\prime}\right)^{*} \longrightarrow H^{1}\left(k_{S}, T\right) \longrightarrow P^{1}\left(k_{S}, T\right), \\
0 \longleftarrow H^{0}\left(k_{S}, T^{\prime}\right)^{*} \longleftarrow P^{2}\left(k_{S}, T\right) \longleftarrow H^{2}\left(k_{S}, T\right) \longleftarrow H^{1}\left(k_{S}, T^{\prime}\right)^{*} \longleftarrow P^{1}\left(k_{S}, T\right)
\end{gathered}
$$

(cf. [5]) and a null sequence :

$$
H^{1}\left(k_{S}, T\right) \longrightarrow P^{1}\left(k_{S}, T\right) \longrightarrow H^{1}\left(k_{S}, T^{\prime}\right)^{*} .
$$


By the equality (1) we conclude the sequence (2) is exact. The passage to infinite $S$ is not difficult.

b) We have an exact sequence $H^{1}\left(k_{S}, F^{v}\right) \rightarrow H^{1}\left(k_{S}, J(F)\right) \rightarrow H^{1}\left(k_{S}, C(F)\right)$, and two isomorphisms $H^{1}\left(k_{S}, J(F)\right) \cong P^{1}\left(k_{S}, F^{\prime}\right)$ (Lemma 2) and $H^{1}\left(k_{S}, C(F)\right)$ $\cong H^{1}\left(k_{s}, F\right)^{*}$ (cf. [5]). Hence the theorem is proved in case $M=F$.

c) Let $M_{J}$ (resp. $\left.M_{C}\right)$ denote the cokernel of $H^{1}\left(k_{S}, J(M)\right) \rightarrow P^{1}\left(k_{S}, M^{\prime}\right)$ (resp. $\left.H^{1}\left(k_{s}, C(M)\right) \rightarrow H^{1}\left(k_{s}, M\right)^{*}\right)$. For any module $A$ of torsion, we put $A(T)=\sum_{p \mid[T]} A(p)$. We get following three commutative exact diagrams :

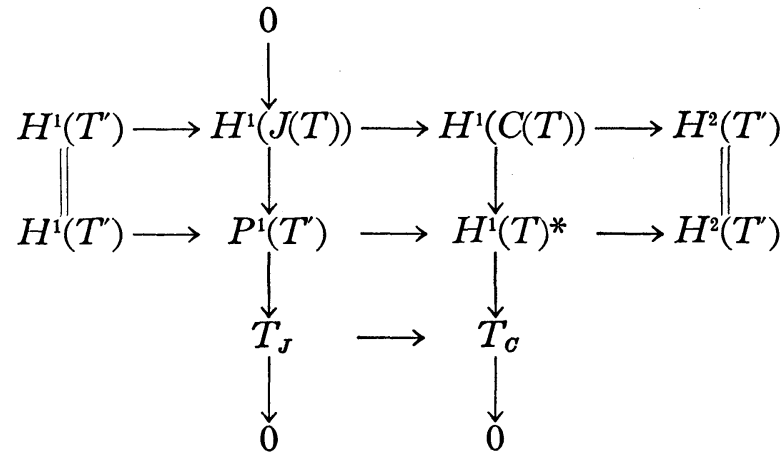

(4)

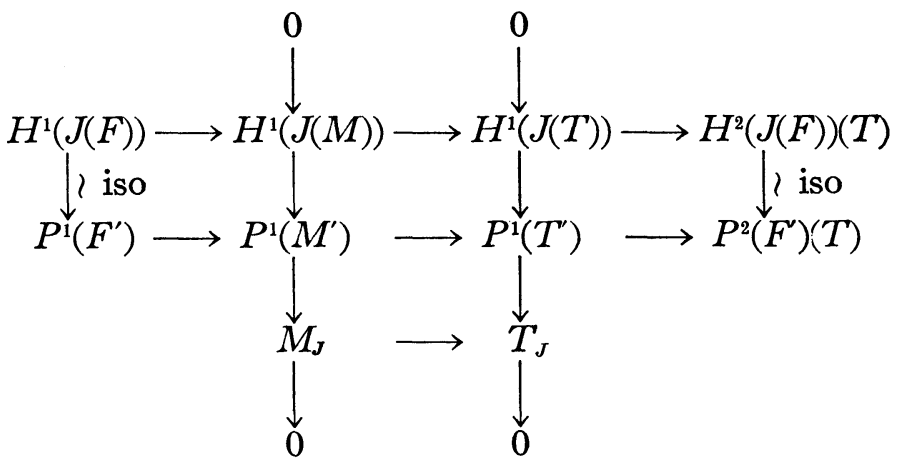

( 5 )

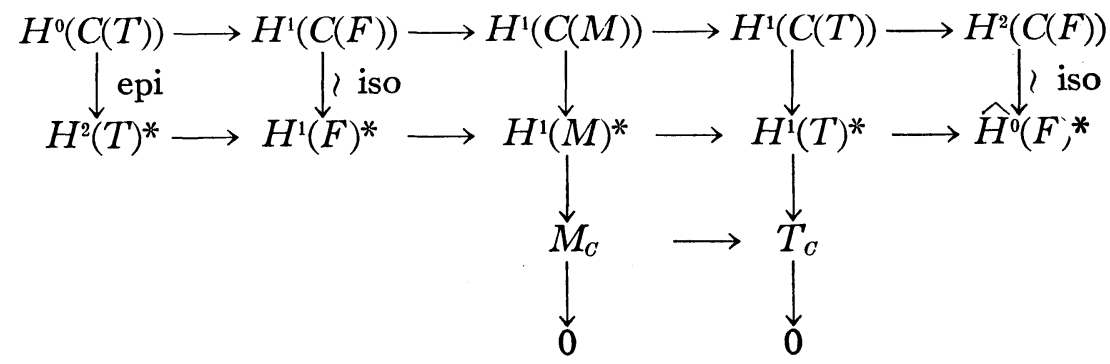


where $H^{r}(\quad)=H^{r}\left(k_{S}, \quad\right)$ and $P^{r}(\quad)=P^{r}\left(k_{S}, \quad\right)$. In the diagram (3), $H^{1}(C(T))$ $\rightarrow H^{1}(T)^{*}$ is necessarily injective, hence $H^{1}(C(M)) \rightarrow H^{1}(M)^{*}$ is also injective by the diagram (5). Since all rows of the above diagrams are exact, we get exact sequences :

$$
0 \longrightarrow T_{J} \longrightarrow T_{C}, 0 \longrightarrow M_{J} \longrightarrow T_{J} \text { and } 0 \longrightarrow M_{C} \longrightarrow T_{C} \text {. }
$$

A commutative diagram

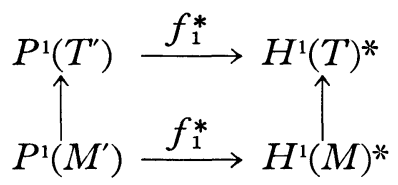

induces a commutative diagram<smiles>[Y10][Te]</smiles>

Hence $M_{J} \rightarrow M_{C}$ is injective. Finally consider a commutative diagram :

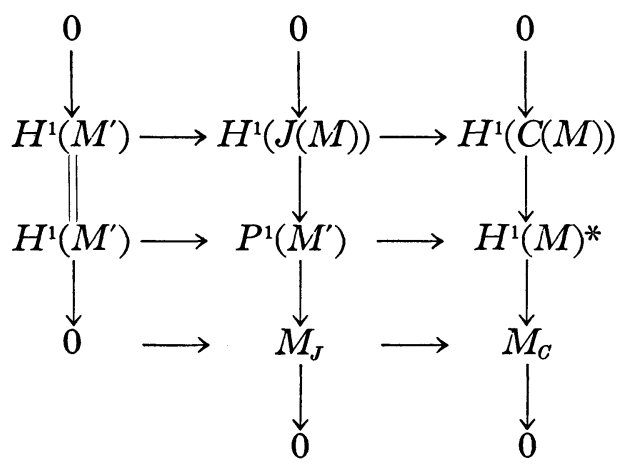

where all sequences are exact except the middle row. Hence the middle row is also exact.

Q.E.D.

REMARK 3. Combining Theorem 4 with [5; Theorem 2], we have an exact sequence

$$
H^{1}\left(k_{S}, M\right) \longrightarrow P^{1}\left(k_{S}, M\right) \longrightarrow H^{1}\left(k_{S}, M^{\prime}\right)^{*} \longrightarrow H^{2}\left(k_{S}, M\right) \longrightarrow P^{2}\left(k_{S}, M\right) .
$$


ADDED IN PROOF: Recently, the author has given the proof of the Tate's assertion in Remark 2. See Proc. Japan Acad., 44(1968), 771-775.

\section{REFERENCES}

[1] A. BRUMER, Galois groups of extensions of algebraic number fields with given ramification, Michigan Math. Journ., 13(1966), 33-60.

[2] J.-P. SERRE, Cohomologie galoisienne, Springer Lecture Series No. 5 (1963).

[3] J. TATE, Duality theorems in Galois cohomology over number fields, Proc. Internat. Congress Math. 1962, Stockholm, 288-295.

[4] J. TATE, On the conjectures of Birch and Swinnerton-Dyer and a geometric analog, Sém. Bourabki, exp. $306(1965 / 66)$.

[ 5 ] K. UchidA, On Tate's duality theorems in Galois cohomology, Tôhoku Math Journ., 21(1969), 92-101.

MATEMATICAL INSTITUTE

TÔHOKU UNIVERSITY

SENDAI, JAPAN 